\title{
Lombalgia: incidência, sintomas e diagnóstico
}

\author{
Gustavo Velloso $^{1}$
}

RESUMO - A dor lombar representa uma queixa habitual feita aos profissionais de saúde em suas diversas áreas de atuação. É importante lembrar que a apresentação da dor lombar pode estar associada à pratica desportiva, profissional, a idade paciente e seu estado geral de saúde. A dor é a queixa mais importante e junto com um criterioso exame clínico deve ser bem avaliada. A utilização de exames complementares para elucidar a causa da lombalgia é feita com bases em achados clínicos objetivos e precisam ser avaliados com critério.

Palavras chave: lombalgia, sintomas, avaliação, exames.

\section{Low back pain: incidence, presentation and diagnosis}

\begin{abstract}
Low back pain represents habitual complain dealt by health professions in their many branches. It is important remember that presentation of pain can be associated to sports activities, professional life, age, and general health of the patient. Pain is by far the most important complain and must be well evaluated. Complementary test used to diagnose the source of low back pain must be carried out after finding of consistent and objective signs.
\end{abstract}

Key words: low back pain, symptoms, evaluation, tests.

A lombalgia constitui problema de saúde pública onipresente. Hoje, é a doença mais comum no homem e só perde a liderança para as altas taxas de resfriado que acometem as diversas faixas etárias da população mundial. (KELSEY et al, 1988) É definida como sensação de dor ou rigidez localizada na extremidade da coluna vertebral, situada acima das nádegas ou como dor secundária ao uso excessivo de estrutura anatômica normal ou, ainda, como dor secundária a trauma ou deformidade de estrutura anatômica.

\footnotetext{
${ }^{1}$ Professor da disciplina de ortopedia da Faculdade de Ciências da Saúde do Centro Universitário de Brasília-UniCEUB

Correspondência: grvelloso@ terra.com.br
} 
Do ponto de vista da epidemiologia, os estudos já realizados pelas autoridades de saúde dos Estados Unidos da América do Norte indicam que a lombalgia e as dores da coluna vertebral em condições crônicas geram limitação da atividade física após a idade de 45 anos e representam a terceira causa mais freqüente de absenteísmo ao trabalho nas pessoas entre a faixa de 45 e 64 anos, com nítida prevalência do sexo masculino. (ROSSIGNOL et al, 1988) Cerca de 70 doenças de origem não mecânica já tiveram seus sintomas apresentados pelos pacientes com quadro de lombalgia. (BORENSTEIN et al, 1995) A maioria dos episódios de dor da lombalgia não incapacita o paciente, mais de 50\% deles melhoram depois de uma semana de tratamento, e $90 \%$ melhoram após oito semanas.

Os fatores de risco incluem características ocupacionais ou psicológicas. As ocupacionais são empregos que envolvam levantamento de peso acima da capacidade do empregado ou trabalho em posições inadequadas. Os pacientes acima de 45 anos de idade apresentam risco duas vezes e meia a mais de ausentaremse do trabalho em decorrência da dor na coluna, quando comparados aos trabalhadores de 24 anos de idade ou menos. (ROSSIGNOL et al, 1988) Condições psicológicas estão também associadas às dores de coluna; a depressão é a complicação mais frequiente segundo Deyo et al (1999). O cigarro e a obesidade também estão associados ao risco de desenvolver dor na região lombar. (NACHENSON, 1976)

A avaliação clínica do paciente com quadro de lombalgia deve ser feita de maneira organizada, ampla e direcionada, em especial, para as queixas principais dos pacientes. Na maioria dos casos, a diversidade das queixas, a dificuldade de aferição da origem dos sintomas e sua correspondência anatômica com a estrutura óssea, ligamentar, muscular, ou discal tornam a identificação da causa da lombalgia um processo difícil. A história clínica que contém informações sobre duração e localização da dor, atividades que precipitam os sintomas e as que a amenizam e a sua irradiação, podem fornecer bases da classificação da lombalgia em dois grupos distintos: a de origem mecânica, caracterizada por períodos de curta duração e associação a tarefas físicas e a de causa secundária, que tende a ter início mais gradual e sem fator precipitante que possa ser identificado. (FRYMOYER et al, 1980)

O profissional de saúde e, em especial, o médico desempenham papel importante na avaliação e no tratamento dos pacientes com queixas de algias da coluna vertebral. A avaliação do comportamento, o exame clínico detalhado e uma postura agressiva e franca na relação entre terapeuta e paciente são importantes para que se entenda que a maioria dos pacientes melhoraram do quadro doloroso e retornam as suas atividades habituais após um período de duas ou três semanas de tratamento. 
O quadro doloroso que domina os sintomas da maioria dos pacientes deve ser diagnosticado e tratado de forma precoce, pois os resultados terapêuticos são mais eficazes e menos onerosos. (TEIXEIRA et al, 2004) A avaliação da dor compreende a quantificação de suas características e suas repercussões biopsicosociais.A forma mais simples de avaliação da intensidade dolorosa é feita pelo uso de escalas analógicas visuais, numéricas, de categorias de expressões verbais e de cores ou objetos, utilizadas de acordo com a população a ser estudada. $\mathrm{Na}$ avaliação da lombalgia é comum dividirmos os tipos de apresentação dolorosa em: dor somática superficial, representada pelos distúrbios que afetam a pele e os tecidos subcutâneos; dor somática profunda, que se origina no eixo da coluna vertebral, músculos adjacentes, tendões, ligamentos e fáscias; dor radicular, que se relaciona ao envolvimento dos nervos vertebrais próximos ao sítio da inflamação ou nos processos que reduzam o fluxo sanguíneo ao nervo; dor neurogênica, que denomina os sintomas decorrentes do envolvimento sensorial de um nervo periférico e pode resultar de anormalidades em órgãos que compartilham a inervação segmentar com a vértebra lombar ou sacral; dor psicogênica, que, por sua vez, não segue padrão dermatogênico e é percebida no córtex cerebral. (TEIXEIRA et $a l, 2004)$

O objetivo do exame físico da coluna lombar e sacra é demonstrar as anormalidades estáticas e dinâmicas que podem auxiliar a descartar doenças responsáveis pelo quadro de lombalgia. É importante salientar que a coluna lombar tem a característica de suportar cargas elevadas em decorrência da superposição do peso do corpo com forças adicionais, como o levantamento de pesos, o ato de saltar, e a postura sentada durante longos períodos. O conjunto anatômico do segmento lombar é constituído por vértebras de morfologia semelhante, músculos com origens e massa variáveis, discos intervertebrais de tamanhos diferentes, mas com conteúdo homogêneo e ligamentos de espessura, tamanho e pontos de inserção bem diferentes. Assim sendo, durante o exame físico, a avaliação do alinhamento da coluna deve ser relacionada com a carga suportada, a mobilidade da coluna com a idade do paciente, o enrijecimento das articulações e a degeneração dos corpos vertebrais com a perda de massa óssea.

Os testes de movimentação e função da coluna lombar podem ser utilizados de acordo com rotina determinada para minimizar o tempo gasto e o desconforto ao qual o paciente é submetido. As anomalias sensoriais e motoras devem ser avaliadas de modo criterioso e ampliadas de maneira mais detalhada se presentes em um ou mais segmentos da coluna examinada. Sabe-se que o exame físico é capaz de acrescentar informações importantes na avaliação dos pacientes com lombalgia, mas a dificuldade em reproduzir os sintomas e a significância dos achados anormais devem ser levadas em consideração sempre que comparamos grupos não homogêneos de pacientes. 
As investigações complementares iniciais em pacientes com quadro de lombalgia nem sempre requerem exame radiológico da coluna lombar ou vertebral. A indicação habitual para estes exames está baseada em histórico de trauma evidente ou quadro agudo constitucional. Os pacientes com lombalgia de origem mecânica apresentam, com freqüência, resultados normais no exame radiológico. (NACHENSON, 1976) É importante lembrar também que, nos adultos com menos de 50 anos de idade, os resultados positivos de radiografias planas podem ser tão baixos quanto 1 em 2500 exames. (EPSTEIN et al, 1987) Nos pacientes com alterações radiológicas ocasionadas por osteoartrite, é comum encontrarmos cerca de dois terços dos pacientes assintomáticos ou que apresentem sinais mínimos de alteração óssea e, assim, torna-se difícil a correlação dos seus sintomas com a lombalgia. (LAURENCE et al, 1986) A recomendação atual feita por Scavone et al (1991) é de pacientes com quadro de lombalgia que apresentem: trauma significativo, câncer prévio, infecção recente, abuso no uso de medicamentos, perda de peso ou dor ao repouso e que sejam avaliados com a utilização de exames radiológicos simples. (GEHWEILER et al, 1993)

A tomografia computadorizada é também indicada na investigação de alguns pacientes com lombalgia. O exame permite o escaneamento da estrutura óssea da coluna vertebral e suas relações com ligamentos, raízes nervosas, planos gordurosos e disco intervertebral. É capaz de delinear as imagens de tumores de partes moles que invadem o tecido ósseo bem como as lesões destrutivas que ocorrem dentro do osso. É importante salientar que a utilização da tomografia computadorizada nos casos de lombalgia deve ser feita com base em dados clínicos que necessitem de confirmação e não como exame complementar, como a radiografia simples.

As imagens geradas pelo o exame de ressonância magnética contribuem de modo decisivo para nova perspectiva na imagenologia da coluna vertebral e sacral. O exame não é invasivo e não expõe o paciente à emissão de radiação, como acontece com a tomografia computadorizada, pois as imagens são decorrentes do uso de ondas magnéticas. A ressonância é útil na avaliação de alterações mecânicas da coluna vertebral e é capaz de produzir imagens com muita nitidez do disco intervertebral e da medula e suas raízes. Estudos prospectivos que utilizam tanto a tomografia computadorizada quanto a ressonância magnética revelaram que os pacientes sem histórico ou achado clínico que indiquem patologia vertebral possuem uma chance em três de apresentarem resultado de exame normal em ambos os exames. (WIESEL et al,1994; JENSEN et al, 1994) Achados anormais na morfologia do disco vertebral são mais confiáveis em pacientes sintomáticos com menos de cinqüienta anos de idade, pois, acima desta faixa etáriam, os achados positivos da ressonância magnética estão relacionados com expressiva incidência de achados degenerativos do segmento lombo-sacral. 
Os exames laboratoriais não fazem parte da rotina de investigações utilizadas para avaliar pacientes com quadro de lombalgia. Exceção é feita aos pacientes com mais de sessenta anos e história clínica indicando alterações no estado geral. Nestes pacientes, o teste de velocidade de hemossedimentação elevado sugere quadro inflamatório tecidual, e as anormalidades no hemograma completo podem levantar a suspeita de neoplasia.Os exames laboratoriais para avaliar taxas de cálcio, fósforo e das fosfatases só devem ser solicitados quando embasados por indicação clínica bem fundamentada.

A confirmação ou exclusão de sinais e sintomas neurológicos associados ao quadro da lombalgia aguda ou crônica tem, nos exames de eletrodiagnóstico, técnica exame que permite identificar com razoável precisão a compressão e ou degeneração das raízes nervosas. A velocidade de condução dos nervos periféricos e a mensuração de atividade elétrica gerada pela contração muscular documentam de forma objetiva as normalidades eletrofisiológicas. Entretanto, devemos considerar que existem importantes limitações dos estudos eletrodiagnósticos na avaliação de pacientes com lombalgia. A duplicidade de enervação de mesmo músculo próximo a uma raiz e a dificuldade de correlacionar os achados positivos de um grupo significativo de pacientes com única queixa são limitações descritas na literatura sobre os exames eletrodiagnósticos. (HALDEMAN et al, 1994)

Assim sendo, devemos conceber a lombalgia como uma patologia do sistema músculo-esquelético que ocorre com freqüência entre os pacientes na idade produtiva. O quadro doloroso é o mais predominante entre outros sintomas. A avaliação criteriosa de cada paciente por meio de um exame clínico detalhado, complementado por exames de imagem quando necessário, pode contribuir de maneira decisiva para esclarecer a origem dos sintomas. A lombalgia deve ser considerada uma patologia de difícil diagnóstico, multifatorial na sua etiologia e que deveria ser estudada com mais frequiência na literatura da área de saúde.

\section{Referências}

BORENSTEIN, D.G.; WIESEL.,W.S. Low back pain: medical diagnosis and comprehensive management. 2E Philadelphia: W.B. Saunders, 181,1995

DEYO,R.A. BASS, J.E. Lifestyle and low back pain. Spine.14:501,1999.

EISEN A. Electrodiagnosis of radiculopathies . Neurologic Clin. 3:494.1995

EPSTEIN ,B.S; EPSTEIN, J.A. Lumbar spinal stenosis .Radiol Clin. North Am. 15: 227,1897.

FRYMOYER, J.W. \& POPE, M.H. Epidemiology studies of low back pain. Spine 5: 419,1980.

GEHWEILER, J.A \& DAFFNER, R.H. Low back pain.AJR. 140: 109,1993. 
HALDEMAN, S. Eletrodiagnostic evaluation of nerve root function .Spinne.9:42, 1994.

JENSEN, MC. \& BRAND MN. Magnectic resonance scans in people without back pain. N.Engl J Med. 69:331.1994

KEWLSEY, J.L. \& WHITE A. Epidemiology of back pain. Spine.5:133,1988.

LAWRENCE.J.S. \& BIER, F. Osteoarthosis: prevalence in the population and relationship between symptoms and xray changes.Ann Rheum Dis. 25: 1,1966.

NACHEMSON, A. The lumbar spine : an orthopaedic challenge. Spine, 1:59,1976.

ROSSIGNOL, M.; SUISSA S.\& ABENHAIM L. Working disability due to occupational back pain.Orthop. Clin North Am, 22:502,1988.

ROSSIGNOL, M. The evolution of compensated occupation spinal injuries.Spine17:1043,1988.

SCAVONE, J.G. LATSHAW, R.F. \& WEIDNER,W.A. Anterior posterior and lateral radiographs : and adequate lumbar spine examination.AJR.136:715,1991.

TEIXEIRA, J.T \& YENG, L. Avaliação da dor em ortopedia. Traumatologia e Ortopedia,05:6, 2004

WIESEL, S.W, TSOURNAS E FEFFER H.L. A study of computer assisted tomography.Spine, 9;549,1994. 